\title{
FP-(L)APW + lo study of mechanical stability and electronic behavior of CoGe in B20 structure
}

\author{
M. A. Timaoui ${ }^{2, *}$, H. Bouafia ${ }^{1,4}$, B. SAhli $^{1}$, S. Hiadsi $^{2}$, B. Abidri ${ }^{3}$, A. BouazA $^{1}$, \\ A. AKriche ${ }^{2}$, D. Kerroum ${ }^{2}$ \\ ${ }^{1}$ Laboratoire de Génie Physique, Université Ibn Khaldoun, Tiaret 14000, Algérie \\ ${ }^{2}$ Laboratoire de Microscope Electronique et Sciences des Matériaux, Université des Sciences et de la Technologie Mohamed \\ Boudiaf, Département de Génie Physique, BP1505 El m'naouar, Oran, Algérie \\ ${ }^{3}$ Laboratoire des Matériaux Magnétiques, Université Djillali Liabés, Sidi Bel-Abbes 22000, Algérie \\ ${ }^{4}$ Département des Sciences et de la Technologie, Faculté des Sciences Appliquées, Université Ibn Khaldoun,
}

Tiaret 14000, Algérie

\begin{abstract}
The aim of this work is a theoretical study of structural, elastic, electronic and thermal properties of CoGe compound in B20 structure using All-electron self-consistent Full Potential Augmented Plane Waves plus local orbital "FP(L)APW + lo" within the framework of Density Functional Theory DFT. GGA-PBEsol is the exchange-correlation potential selected in this work. This choice is motivated by the success of this functional in predicting structural and mechanical properties of solids. The values obtained by the study of structural properties are in very good agreement with those found previously. In this work, the elastic constants have been predicted for the first time and the obtained values confirm the mechanical stability of the CoGe compound in its B20 structure. The electronic part of this work shows that CoGe has metallic behavior with a mixed bonding between cobalt and germanium of covalent-metallic type. The effect of temperature and hydrostatic pressure on the lattice parameter $-\mathrm{a}_{0}$, heat capacity at constant volume $-\mathrm{C}_{\mathrm{V}}$, thermal expansion coefficient $-\alpha$ and entropy $-\mathrm{S}$ of the CoGe have been studied using Debye model.
\end{abstract}

Keywords: CoGe; DFT; FP-(L)APW + lo; GGA-PBEsol; mechanical stability; thermal properties.

\section{Introduction}

For over half a century, B20-type compounds have been the subject of intensive research due to their important physical properties [1, 2]. Because of their interesting magnetic properties, these materials have been recognized to be of strategic importance for spintronics applications [3]. Quite naturally, B20-type compounds are designed to be intrinsically compatible with the established silicon technologies. They have been proven to be effective and also innovative for spin injection into silicon devices [4].

Co-Ge compounds are ready to master the challenges of advanced thin films and may become a key factor for the development of all spintronics devices and products that are especially

*E-mail: Abdeldjalil.timaoui@univ-usto.dz influential in local magnetization of semiconductor matrix, redistribution of electron density in alloys and so on [5].

Because of its high crystalline anisotropy and low recording noise there are many relevant areas of CoGe applications with no interference with the consumers' interest in environmentally friendly products as well as with the respective regulations [1]. These areas include the biological impact of electromagnetic emission, electromagnetic interference, energy conservation and emission as well as public health, especially prevention of hazards caused by electromagnetic emission systems [6]. Certain B20 often display interesting magnetic, thermal and transport properties despite the fact that the possibility of their corresponding phase transformation is problematic [7-9].

A thermoelectric material capable of converting waste heat into electricity or pump heat, such as 
a Peltier cooler is a candidate for a sustainable energy solution. In our daily lives, we use high efficiency materials. Developing thermoelectric highly effective materials is one of the most demanding goals in material science [10].

CoGe belongs to the most promising thermoelectric materials due to its low resistivity and high thermal power. Concerning the properties of such compounds, research is still needed [10].

A recent study presenting magnetization, specific heat, resistivity, and Hall effect measurements on the cubic B20 phase of CoGe has shown that this is a low carrier density metal with a very small, nearly temperature-independent diamagnetic susceptibility [4]. Several Co-Ge compounds have been the subject of a reasonable description by first principle calculations before optimization to determine their enthalpies of formation [5]. Experimentally, X-ray diffraction technique has been used to investigate the crystalline structures of some $\mathrm{Co}-\mathrm{Ge}$ alloys $\left(\mathrm{Co}_{\mathrm{x}} \mathrm{Ge}_{100-\mathrm{x}}\right)$ [6].

It is however interesting to note that for Co some researchers have reported first phase formation of CoGe [11]. In germanides, incongruent phases with more negative effective heat of formation have been found occasionally as first phases; well-known examples include $\mathrm{CoGe}$ in the $\mathrm{Co}-\mathrm{Ge}$ system [12].

The aim of this study is to confirm theoretically the results already achieved in other works, including the experimental ones, to highlight the performance and efficiency of the method used in this work (FP-(L)APW + lo) [13, 14]. A further study is also done to predict some properties which are still unknown for CoGe in its B20 structure, especially the elastic constants and the related parameters. This study also includes the analysis of electron density by the theory of atom in molecule QTAIM [15-18].

The crystal structure of CoGe compound is cubic, of space group $\mathrm{P} 2{ }_{1} 3$ where cobalt and germanium atoms are located according to the following coordinates: $(\mathrm{u}, \mathrm{u}, \mathrm{u}),(0.5+\mathrm{u}, 0.5-\mathrm{u},-\mathrm{u}),(-\mathrm{u}$, $0.5+\mathrm{u}, 0.5-\mathrm{u})$ and $(0.5-\mathrm{u},-\mathrm{u}, 0.5+\mathrm{u})$ [4] as shown in Fig. 1 obtained using XCrySDen package [19].

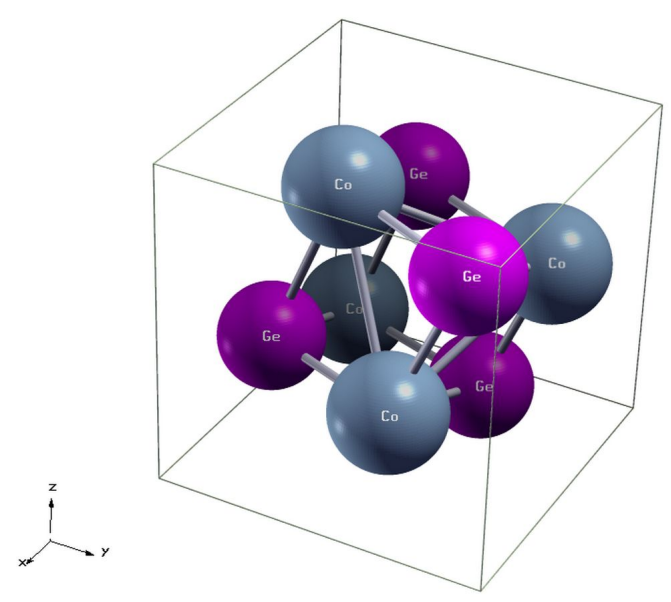

Fig. 1. Representation of CoGe B20 crystal structure.

This paper can be divided into three basic parts: Section 2 paves the way to the next part, it is a complete overview and detailed description of the computational method designed for the study. Section 3 includes the most relevant results. The last section contains conclusions that reflect the objectives and the challenges of the future.

\section{Computational method}

FP-(L)APW + lo method is one of the most important powerful tools that aroused great interest in the field of theoretical prediction of physical properties of solid materials including structural, mechanical and electronic [20]. This success is mainly due to the theoretical advancement including new functional and potential [21, 22] (e.g. GGA-PBEsol to predict structural and mechanical properties of solid materials). Thanks to these functional, solving Kohn and Sham equations became possible in the frame of DFT [23]. The obtained results are generally satisfactory within acceptable accuracies with the experimental values [24].

This theoretical model is implemented in a large number of codes but from the point of view of performance and efficiency, WIEN2k code [25, 26] has been proven the most efficient in the field of physics. 


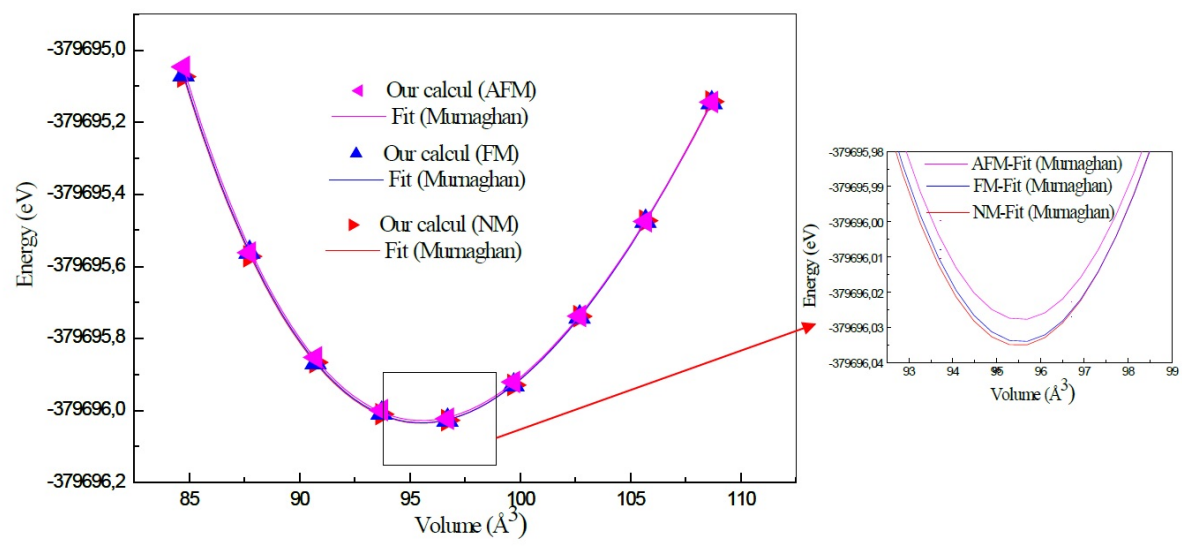

Fig. 2. Variation of total energy versus volume curve for nonmagnetic (NM), antiferromagnetic (AFM) and ferromagnetic (FM) states of CoGe in B20 crystal structure.

Regarding the basic function, we have selected $\mathrm{R}_{\mathrm{MT}} \cdot \mathrm{K}_{\mathrm{MAX}}=8.5$, where $\mathrm{R}_{\mathrm{MT}}$ represents the smallest radius of an atomic sphere within which the wave functions are a basic set spherical harmonics around the nuclei and $\mathrm{K}_{\mathrm{MAX}}$ represents the magnitude of the largest $\mathrm{K}$ vector in the plane wave expansion. Similarly, we have adopted an optimum value of $\mathrm{I}_{\max }=10$ for partial waves inside the atomic spheres, while the Fourier charge density has been increased to $\mathrm{G}_{\max }=12 \mathrm{bohr}^{-1}$. We developed $\mathrm{k}$ mesh integration points of the Brillouin zone [27] in height of $(12,12,12)$ grid producing $84 \mathrm{k}$-points in the irreducible Brillouin zone (IBZ). In our calculations, we have chosen for the CoGe compound the muffin-tin radii equal to 2.1 and 2.0 bohr for Co and Ge, respectively. When the total energy converges to a value less than the chosen value of $10^{-4} \mathrm{eV}$, the self-consistent calculations also converge.

\section{Results and discussions}

\subsection{Structural properties}

To study the mechanical stability as well as electronic and thermodynamic properties of $\mathrm{CoGe}$ using the FP-(L)APW + lo method, structural properties must be first predicted in order to confirm that the non-magnetic phase is the most stable. In addition, different structural parameters necessary for calculation of other properties should be also obtained. For this purpose, we studied the variation of total energy of the unit cell volume of different magnetic phases (non-magnetic, ferromagnetic and antiferromagnetic) in the vicinity of the equilibrium lattice parameter $\mathrm{a}_{0}$ whose energy corresponds to that of the ground state (Fig. 2). Different structural results can be obtained by fitting different curves $\mathrm{E}(\mathrm{V})$ using the well-known Murnaghan equation of state [28] given by:

$$
E(V)=E\left(V_{0}\right)+\frac{B_{0} V}{B^{\prime}}\left[\frac{\left(\frac{V_{0}}{V}\right)^{B^{\prime}}}{B^{\prime}-1}+1\right]-\frac{B_{0} V_{0}}{B^{\prime}-1}
$$

where $\mathrm{B}_{0}$ and $\mathrm{B}^{\prime}$ are the bulk modulus at equilibrium and its pressure derivative, respectively, $V_{0}$ is the volume of the unit cell at equilibrium. The lattice parameter $\mathrm{a}_{0}$ corresponding to the ground state is deduced from the minimum of the curve $\mathrm{E}_{\text {tot }}(\mathrm{V})$.

The obtained results are summarized in Table 1. It is noted that the lowest total energy is that of the non-magnetic phase which confirms the information already obtained for this compound [4]. For the lattice parameter $\mathrm{a}_{0}$, it is to be noted that the calculated values are very close to those found experimentally and theoretically. The same applies to the calculated internal parameters $\left(\mathrm{U}_{\mathrm{Co}}\right.$ and $\left.\mathrm{U}_{\mathrm{Ge}}\right)$; the obtained results are almost similar to other available works which confirms the success of the GGA-PBEsol functional 
in predicting structural properties of solid materials. The results for the bulk modulus are similar to those found, however, there is a lack of theoretical and experimental results for the ground state energy and the cohesive energy of this compound.

\subsection{Mechanical properties}

The study of elastic properties of a solid material is very important, because it allows predicting its mechanical stability in a given structure, and deducing several physical quantities related to mechanical properties of materials, in particular Young's modulus, Poisson ratio and Debye temperature.

For a cubic material, due to its high symmetry, there are only three elastic constants, denoted as $\mathrm{C}_{11}, \mathrm{C}_{12}$ and $\mathrm{C}_{44}$. Knowledge of the values of these constants allows us to predict the mechanical stability of any cubic material through the following relationship [29]:

$$
\begin{aligned}
& C_{11}-C_{12}>0, C_{11}>0, C_{44}>0, \\
& C_{11}+2 C_{12}>0, C_{12}<B<C_{11}
\end{aligned}
$$

Note that, so far, no results have been available for the elastic constants of CoGe-compound in its B20 structure, which gave us the opportunity to contribute in the scientific literature by the first prediction of elastic properties for this compound.

Several theoretical models can be used to predict the elastic constants by evaluating the total energy of a unit cell under slight strain effect. In this work, to provide maximum information on the elasticity of CoGe compound, we have used two models that are implemented in the IRelast package provided by Jamal et al. [30, 31] which is compatible with the Wien2k package and that of Mehl [32]. These models have been proven to be effective because they have already been tested in many previous works.

In IRelast package method, three strains are applied: $\mathrm{D}_{1}$ (orthorhombic distortion with preserved volume), $\mathrm{D}_{2}$ (the same constraint is applied to the three directions while preserving cubic symmetry of material but without conservation of volume) and $\mathrm{D}_{3}$ (a monoclinic distortion with preserved volume) to calculate respectively $\left(\mathrm{C}_{11}-\mathrm{C}_{12}\right)$, $\left(\mathrm{C}_{11}+2 \mathrm{C}_{12}\right)$ and $\mathrm{C}_{44}$.

Different values of elastic constants may be obtained by a second order polynomial fit of the curves obtained from variation of the total energy under the effect of different strains as is shown in Fig. 3 and by the following relationships:

$$
\begin{aligned}
E_{D 1}(\delta)= & E(0)+V_{0}\left[\left(C_{11}-C_{12}\right) \delta^{2}+O\left(\delta^{4}\right)\right] \\
E_{D 2}(\delta)= & E(0)+V_{0} \delta\left[\tau_{1}+\tau_{2}+\tau_{3}\right] \\
& +V_{0}\left[(2 / 3)\left(C_{11}+2 C_{12}\right) \delta^{2}+O\left(\delta^{3}\right)\right]
\end{aligned}
$$

$$
E_{D 3}(\delta)=E(0)+V_{0}\left[\left(2 C_{44}\right) \delta^{2}+O\left(\delta^{4}\right)\right]
$$

where: $V_{0}$ is the volume of the unconstrained unit cell, $\mathrm{E}(0)$ its corresponding energy and $\tau_{\mathrm{i}}$ are parameters related to the strain of the crystal.

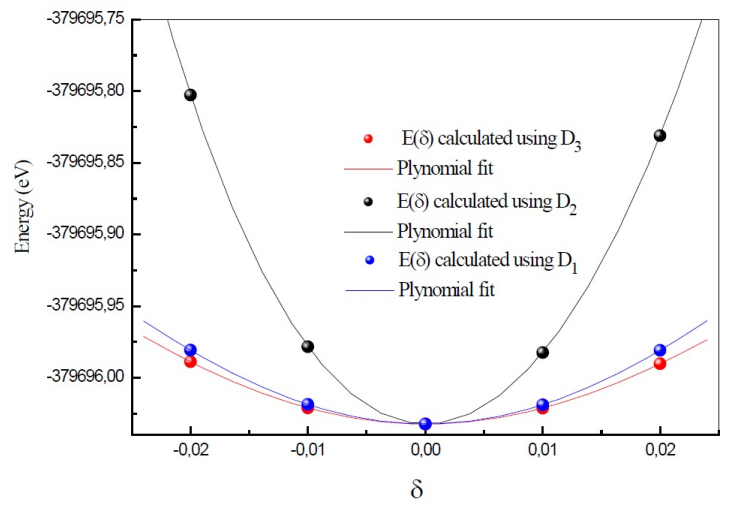

Fig. 3. Total energy as a function of orthorhombic $D_{1}$, cubic $\mathrm{D}_{2}$ and monoclinic $\mathrm{D}_{3}$ strains of CoGe obtained using the implemented model in the IRelast package.

In Mehl method, elastic constants calculation is based on the application of two strains: one orthorhombic to calculate $\mathrm{C}_{11} \mathrm{C}_{12}$ and another monoclinic to calculate $\mathrm{C}_{44}$ by linear fitting of two variation curves in the total energy of the unit cell according to the squares of the strain (Fig. 4) w hich are respectively given by the following relationships:

$$
E(\delta)=E(0)+\left(C_{11}-C_{12}\right) V_{0} \delta^{2}
$$


Table 1. Calculated lattice parameter $\mathrm{a}_{0}[\AA ̊]$, bulk modulus $\mathrm{B}_{0}[\mathrm{GPa}]$ and its pressure derivative $\mathrm{B}^{\prime}$, total unit cell energy $\mathrm{E}_{0}[\mathrm{eV} / \mathrm{cell}]$, cohesive energy $\mathrm{E}_{\mathrm{coh}}[\mathrm{eV} / \mathrm{cell}]$, cobalt internal parameter $\mathrm{U}_{\mathrm{Co}}$ and germanium internal parameter $\mathrm{U}_{\mathrm{Ge}}$ of $\mathrm{CoGe}$ compound, compared to some experimental and other theoretical works.

\begin{tabular}{llllllll}
\hline $\mathrm{CoGe}$ & $\mathrm{a}_{0}$ & $\mathrm{~B}_{0}$ & $\mathrm{~B}^{\prime}$ & $\mathrm{E}_{0}$ & $\mathrm{E}_{\mathrm{coh}}$ & $\mathrm{U}_{\mathrm{Co}}$ & $\mathrm{U}_{\mathrm{Ge}}$ \\
\hline \hline $\mathrm{NM}$ & 4.5717 & 200.2981 & 4.5443 & $-27918.826049-44.3580$ & 0.1374 & 0.8393 \\
$\mathrm{FM}$ & 5.5718 & 200.1073 & 4.6566 & $-27918.825971-44.3569$ & 0.1371 & 0.8392 \\
$\mathrm{AFM}$ & 4.5716 & 200.6011 & 4.9790 & $-27918.825520-44.3508$ & 0.1370 & 0.8394 \\
\hline \multirow{2}{*}{ Experiment } & $4.637[2]$, & & & & $0.140[4]$ & $0.841[4]$ \\
& $4.631[3]$ & & & & & $0.140[10]$ & $0.845[10]$ \\
\multirow{2}{*}{$\begin{array}{l}\text { Other } \\
\text { studies }\end{array}$} & $4.63[10]$, & & & & & & \\
& $4.4680[8]$, & $173.3506[9]$ & $4.9973[9]$ & & & & \\
\hline
\end{tabular}

$$
E(\boldsymbol{\delta})=E(0)+1 / 2\left(C_{44}\right) V_{0} \delta^{2}
$$

These two strains cause a change in the lattice parameter in three directions but they preserve the equilibrium volume.

Table 2 contains different values of elastic constants calculated by both models. To our knowledge, the values of elastic constants for the $\mathrm{CoGe}$ compound in the B20 structure are unknown to the scientific community.

Taking into account the values of elastic constants calculated by the two models according to the stability criteria of a cubic material at zero pressure (equation 2), we can conclude that $\mathrm{CoGe}$ compound is stable mechanically in its B20 structure. Moreover, we can note that the values obtained by both models are similar.

The knowledge of elastic constants allows us to deduce several important mechanical quantities, such as Young's modulus E, shear modulus $\mathrm{G}$ and Poisson's ratio $v$, using the following equations [33-35]:

$$
\begin{gathered}
E=\frac{9 B G}{3 B+G} \\
G=\frac{C_{11}-C_{12}+3 C_{44}}{5} \\
v=\frac{3 B-E}{6 B}
\end{gathered}
$$

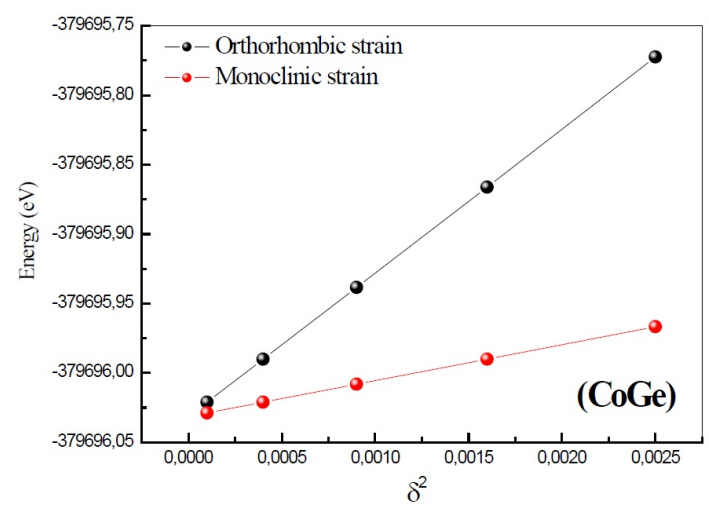

Fig. 4. Total energy as a function of squared orthorhombic and monoclinic strains of CoGe obtained using Mehl method.

The results obtained by the theoretical model implemented in IRelast package and that of Mehl concerning Young's modulus E, shear modulus G and the Poisson's ratio $v$ for CoGe compound are similar to each other. These results are original because of their lack in the literature. The calculated values for Poisson's ratio are close to 0.3 [36] which indicates that $\mathrm{CoGe}$ has a metallic behavior.

Pugh [37] proposed a way to study the ductility and brittleness of a solid material by calculating the $\mathrm{B} / \mathrm{G}$ ratio. According to them, a material is brittle if the $\mathrm{B} / \mathrm{G}$ ratio is less than a limit value of 1.75 and is ductile if it is greater than this limit value. The $\mathrm{B} / \mathrm{G}$ ratio for CoGe was calculated on the basis of the results obtained by both models (Table 2) indicating that $\mathrm{CoGe}$ material is ductile in the B20 structure. 
Anisotropic solid material is characterized by invariance of physical properties along three directions. The elastic constants allow us to study this property with the anisotropy factor A. If the value of this factor is close to 1 , it indicates that the material is isotropic while it is anisotropic for a value different than 1 . The relationship characterizing these two properties, for a cubic material, is given by [38]:

$$
A=2 C_{44} /\left(C_{11}-C_{12}\right)
$$

For the anisotropy factor, the values calculated by the two models are similar and close to unity indicating that $\mathrm{CoGe}$ compound in its B20 structure is isotropic (Table 2).

The knowledge of elastic constants allows predicting a very important physical property that is Debye temperature $\theta_{\mathrm{D}}$ which represents the ability to capture thermal energy from a solid material. It also gives an idea about hardness of the material. Using the elastic model, it is possible to predict this property by using the following equation [38]:

$$
\theta_{D}=\frac{h}{k}\left[\frac{3 n}{4 \pi}\left(\frac{N_{a} \rho}{M}\right)\right]^{1 / 3} V_{m}
$$

where $\mathrm{h}$ represents Planck constant, $\mathrm{k}$ - Boltzmann constant, $n$ - number of atoms per formula unit, $\mathrm{N}_{\mathrm{a}}$ - Avogadro number, $\mathrm{M}$ - molecular mass per formula unit, $\rho$ - density and $\mathrm{V}_{\mathrm{m}}$ - average wave velocity. The later can be established using the relation [39]:

$$
V_{m}=\left[\frac{1}{3}\left(\frac{2}{V_{t}^{3}}+\frac{1}{V_{l}^{3}}\right)\right]^{-1 / 3}
$$

where $V_{1}$ stands for velocity of longitudinal elastic wave, while $V_{t}$ represents velocity of transverse elastic wave. The relationships used to determine these velocities are given by [40]:

$$
V_{l}=\left(\frac{3 B+4 G}{3 \rho}\right)^{1 / 2}
$$

and:

$$
V_{t}=\left(\frac{G}{\rho}\right)^{1 / 2}
$$

Using the Debye model, the calculation of the Debye temperature value gives us the ability to calculate other vibrational quantities, especially the Debye temperature for acoustic phonons $\theta_{\mathrm{a}}$ [41] and the maximum frequency of vibration of solid atoms upon thermal transfer called Debye frequency $\omega_{\mathrm{D}}$ [42] which can be determined by the following relationships:

$$
\omega_{D}=\frac{h}{k_{B}} \theta_{D} n^{-1 / 3}
$$

and:

$$
\theta_{a}=\theta_{D} n^{-1 / 3}
$$

Unfortunately, the values of the Debye temperature, Debye frequency and Debye temperature for acoustic phonons are unknown to the scientific community. On the other hand, the values of these quantities calculated by the two models used in this work (Table 3) are similar to each other, which indicates their authenticity, and we hope will be confirmed by additional future work.

\subsection{Electronic properties}

The study of electronic properties provides information on the electronic behavior of a material, on the type of bonds between the atoms that form it as well as different electronic states constituting the valence band responsible for most of optical and electrical properties.

According to the band structure presented in Fig. 5 for CoGe compound in B20 structure, we notice a slight band overlapping between the top of the valence band and the bottom of conduction band which allows stating that this material has a semimetallic behavior, confirming the results already obtained by other published work [8].

To investigate the contribution of different states forming the valence and conduction bands, we traced the curves of calculated partial and total density of states of the top of valence band and those of the bottom of conduction band. From Fig. 5, we can clearly see that the top of valence band is formed essentially by $\mathrm{d}$ states of cobalt with low contributions of $\mathrm{d}$ states of germanium 
Table 2. Calculated elastic constants $\mathrm{C}_{11}$ [GPa], $\mathrm{C}_{12}$ [GPa], $\mathrm{C}_{44}$ [GPa], Young modulus $\mathrm{E}$ [GPa], shear modulus $\mathrm{G}$ [GPa], Zener anisotropy factor A, Poisson ratio $v$ and $\mathrm{B} / \mathrm{G}$ ratio for $\mathrm{CoGe}$.

\begin{tabular}{lcccccccc}
\hline & $\mathrm{C}_{11}$ & $\mathrm{C}_{12}$ & $\mathrm{C}_{44}$ & $\mathrm{E}$ & $\mathrm{G}$ & $\mathrm{A}$ & $v$ & $\mathrm{~B} / \mathrm{G}$ \\
\hline \hline Mehl method & 315.993 & 142.451 & 86.679 & 227.340 & 86.715 & 0.999 & 0.3108 & 2.3098 \\
IRelast package method & 320.473 & 142.420 & 107.144 & 257.238 & 99.897 & 1.204 & 0.2875 & 2.0198 \\
Other results & - & - & - & - & - & - & - & - \\
\hline
\end{tabular}

Table 3. Calculated longitudinal, transverse, average sound velocity $\mathrm{v}_{\mathrm{l}}, \mathrm{v}_{\mathrm{t}}, \mathrm{v}_{\mathrm{m}}\left[\mathrm{m} \cdot \mathrm{s}^{-1}\right]$, Debye temperature $\theta_{\mathrm{D}}[\mathrm{K}]$, Debye temperature for an acoustic phonon $\theta_{\mathrm{a}}[\mathrm{K}]$ and Debye frequency $\mathrm{w}_{\mathrm{D}}[\mathrm{Hz}]$ for CoGe.

\begin{tabular}{lcccccc}
\hline & $\mathrm{V}_{\mathrm{l}}$ & $\mathrm{V}_{\mathrm{t}}$ & $\mathrm{V}_{\mathrm{m}}$ & $\theta_{\mathrm{D}}$ & $\theta_{\mathrm{a}}$ & $\mathrm{w}_{\mathrm{D}}$ \\
\hline \hline Mehl method & 5877.1461 & 3079.12599 & 3444.07488 & 448.4503 & 224.2251 & $4.6720910^{12}$ \\
IRelast package method & 6051.7274 & 3304.87140 & 3685.68798 & 479.9105 & 239.9552 & $4.9998510^{12}$ \\
Other results & - & - & & - & - & - \\
\hline
\end{tabular}

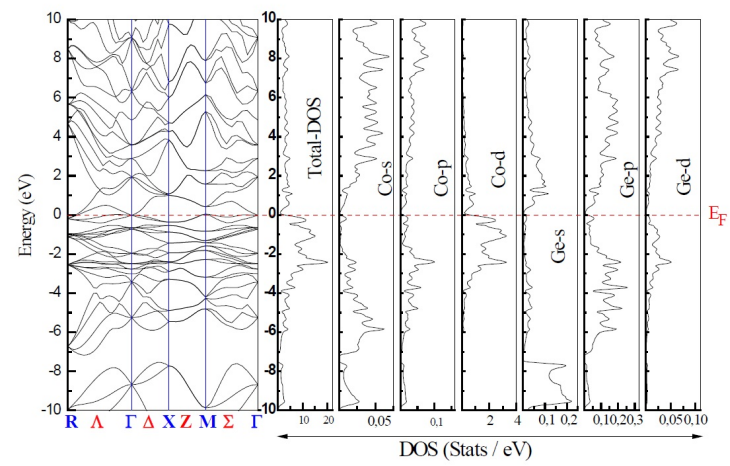

Fig. 5. Calculated band structure, partial and total density of states of CoGe-compound.

and $\mathrm{p}$ states of cobalt and germanium. This indicates hybridization between the states of cobalt and germanium which indicates the existence of a covalent bond between the two atoms of this compound.

To qualitatively analyze the type of bonding between the cobalt and germanium, we have plotted the charge density plots along [ $\left[\begin{array}{lll}1 & 1 & 0\end{array}\right]$ direction of the CoGe compound (Fig. 6). The nonspherical shape of the germanium contours and the presence of charges in the interstitial region indicate a mixed metal-covalent behavior for the bond between the atoms of this compound. To confirm this conclusion qualitatively, the ionicity factor and topological analysis of charge density using QTAIM (Bader's quantum theory of atoms in

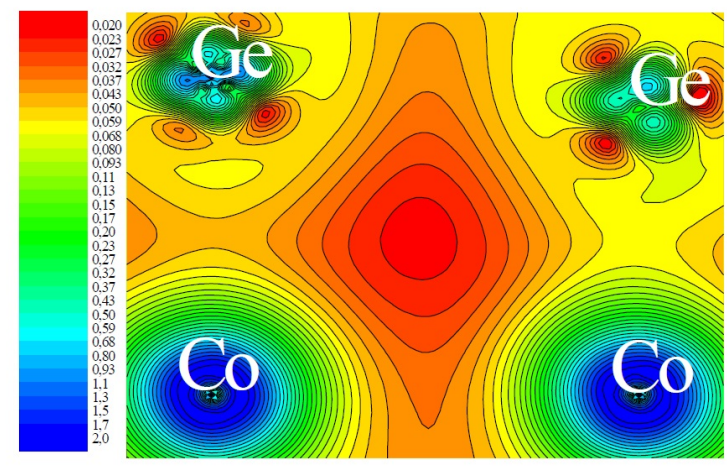

Fig. 6. Calculated charge density along the [110] direction of CoGe-compound; (a) Germanium-basin, (b) Cobalt-basin

molecules) can be used as an efficient way for this kind of study.

According to Pauling rule [43], the calculation of the iconicity factor $F_{i}$ for a solid material is based on electronegativity of the elements forming this compound. The iconicity factor for the bond between two atoms A and B can be calculated by:

$$
F_{i}=1-\exp \left\{-\frac{\left(\chi_{A}-\chi_{B}\right)^{2}}{4}\right\}
$$

where $\chi_{\mathrm{B}}$ and $\chi_{\mathrm{A}}$ represent the electronegativities of $\mathrm{A}$ and $\mathrm{B}$ atoms, respectively.

A $F_{i}$ value close to 1 indicates that the bond is ionic and a value close to 0.5 indicates that the bond is polar, while a value close to 0 indicates that 
the bond is covalent. According to Table 4, the calculated value of the iconicity factor for the bond between cobalt and germanium is about $\sim 0.0042$ which is a very small value, close to zero, which confirms the existence of strong covalent bonding between these two atoms.

Topological analysis of charge density using Bader's quantum theory of atoms in molecules (QTAIM) also allows the prediction of the predominant bond in a solid material or a molecule. This is done by calculating the degree of ionicity $\alpha$ which is based on the topological charge of $\Omega$ atomic basin $\mathrm{Q}(\Omega)$ and the nominal oxidation state of $\Omega$ atom $-\mathrm{O}_{\mathrm{D}}(\Omega)$ (the shapes of the atomic basin for cobalt and germanium are presented in Fig. 7). Its expression is given by $[17,18]$ :

$$
\alpha=\frac{1}{N} \sum_{\Omega=1}^{N} \frac{Q(\Omega)}{O_{D}(\Omega)}
$$

According to the (QTAIM) theory and the relationship mentioned above, a factor $\alpha$ close to 1 means that the bonding of a studied compound is in a large part ionic while a factor near zero indicates the predominance of covalent bond. If the value of this factor is between 0.3 and 0.6 , it means that we have a polar bonding.

According to Table 4, the degree of ionicity $\alpha$ calculated by Bader quantum theory of atoms in molecules (QTAIM) is very close to zero indicating the predominance of the covalent bond between cobalt and germanium, which confirms the previous conclusions.

It remains to confirm the semimetallic behavior of this compound by calculating the degree of metallicity called electron density flatness expressed as [44]:

$$
f_{\Omega}=\frac{\rho_{c}^{\min }}{\rho_{b}^{\max }}
$$

where $\rho_{\mathrm{c}}^{\min }$ represents the absolute minimum of electron density in the interstitial region (called a cage critical point of $\rho$ ), and $\rho_{\mathrm{b}}^{\max }$ represents the maximum value of electron density found among the bond critical points. We know that a $\mathrm{f}_{\Omega}$ value close to one indicates a strong metallic character

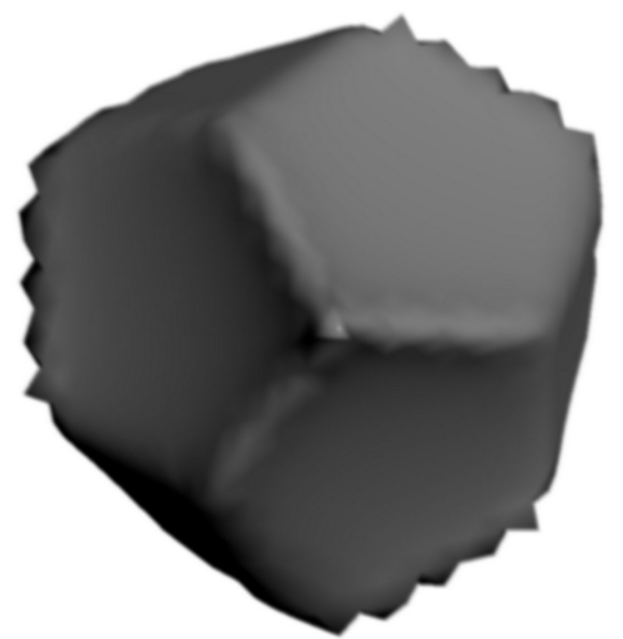

(a) Germanium-basin

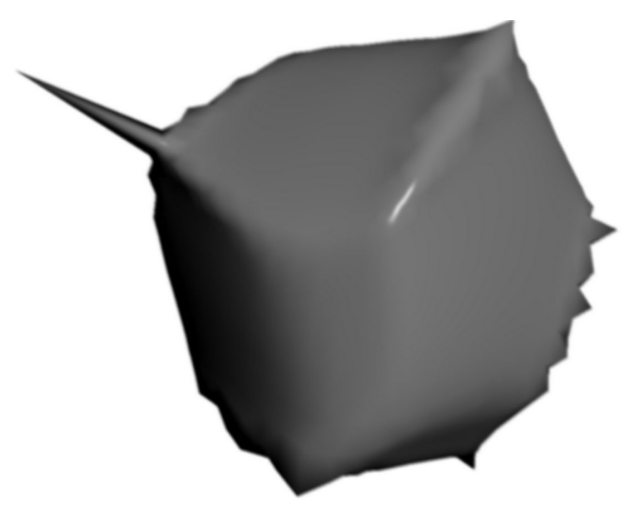

(b) Cobalt-basin

Fig. 7. 3D representations of atomic basins for cobalt and germanium.

while a value close to zero indicates no-metallicity of studied material. The found value for the $\mathrm{CoGe}$ indicates the metallic behavior for this compound with a rate/value of $\sim 0.31$, which means that $\mathrm{CoGe}$ compound has not a fully metallic behavior. This absolutely confirms the previous findings and allows us to say that the bonding between the atoms of the CoGe compound in B20 structure is mixed, it is metallic-covalent and $\mathrm{CoGe}$ is a semimetal material.

\subsection{Thermal properties}

Thermodynamic properties of a material characterize the behavior of the material and vibrations of atomic lattice under the effect of heat energy it absorbs. The Debye model [45] is well known 
Table 4. Calculated atomic basin volume $\left(\mathrm{V}_{\Omega}\right)$, topological charge $\left(\mathrm{Q}_{\Omega}\right)$, degree of ionicity $(\alpha)$, iconicity factor $\left(\mathrm{F}_{\mathrm{i}}\right)$ and factor of electron density flatness $\left(\mathrm{f}_{\Omega}\right)$ for CoGe compound.

\begin{tabular}{lccccc}
\hline & $\mathrm{V}_{\Omega}\left[\AA^{3}\right]$ & $\mathrm{Q}_{\Omega}$ & $\alpha$ & $\mathrm{F}_{\mathrm{i}}$ & $\mathrm{f}_{\Omega}$ \\
\hline \hline $\mathrm{CoGe}$ & & & 0.000056975 & 0.004216087 & 0.310676 \\
\hline $\mathrm{Co}$ & 11.193757 & -0.1760862 & & & \\
$\mathrm{Ge}$ & 12.693875 & 0.1763141 & & & \\
\hline
\end{tabular}

and suitable to calculate several thermodynamic quantities such as variation of the lattice parameter $-\mathrm{a}_{0}$, heat capacity at a constant volume $-\mathrm{C}_{\mathrm{V}}$, thermal expansion $-\alpha$ and entropy $-\mathrm{S}$ under the effect of temperature and hydrostatic pressure. This can be achieved by minimization of the Gibbs energy given by [46-50]:

$$
G *(V, P, T)=E(V)+P V+A_{v i b}\left[\theta_{D}(V), T\right]
$$

where $\mathrm{P}$ is the applied hydrostatic pressure, $\mathrm{V}$ is the unit cell volume at a given pressure and temperature, $E(V)$ is the unit volume energy, $A_{\text {vib }}$ is the vibrational Helmholtz free energy and $\theta_{D}(V)$ is the Debye temperature at a given unit cell volume.

The relationship of the minimization of the Gibbs energy is given at a constant volume and temperature, its expression is given by:

$$
\left[\frac{\partial G^{*}(V ; P ; T)}{\partial V}\right]_{P, T}=0
$$

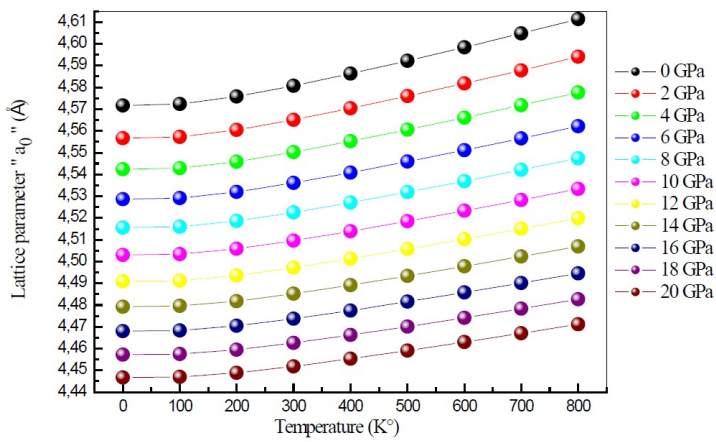

Fig. 8. Variation of the lattice parameter $\mathrm{a}_{0}$ as a function of temperature for $\mathrm{CoGe}$ at different pressures.

By solving equation 22, we can directly get the heat equation (EOS) that allows us to deduce the effect of hydrostatic pressure and temperature on the volume of the solid $-\mathrm{V}$, its thermal capacity $-\mathrm{C}_{\mathrm{V}}$, the isotropy $-\mathrm{S}$ and the coefficient of thermal expansion $-\alpha$ which are given by the following relationships [51]:

$$
C_{V}=3 n k\left[4 D\left(\frac{\theta_{D}}{T}\right)-\frac{3 \theta_{D} / T}{e^{\theta_{D / T}-1}}\right]
$$

and:

$$
S=n k\left[4 D\left(\frac{\theta_{D}}{T}\right)-3 \ln \left(1-e^{-\theta_{D} / T}\right)\right]
$$

and:

$$
\alpha=\frac{\gamma C_{V}}{B_{T} V}
$$

where the Grüneisen parameter $\gamma$ is given by:

$$
\gamma=-\frac{d \ln \theta_{D}(V)}{d \ln V}
$$

The main purpose of this paper is to study the effect of hydrostatic pressure and temperature on the lattice parameter $-\mathrm{a}_{0}$, the heat capacity $-\mathrm{C}_{\mathrm{V}}$, isotropy $-\mathrm{S}$ and the thermal expansion coefficient $-\alpha$ and their values at room temperature for $\mathrm{CoGe}$ compound in its B20 structure.

The effect of temperature and hydrostatic pressure on the unit cell volume and, hence, the lattice parameter of CoGe compound was investigated in the temperature range of $0 \mathrm{~K}$ to $800 \mathrm{~K}$ under a hydrostatic pressure between $0 \mathrm{GPa}$ and $20 \mathrm{GPa}$ (Fig. 8).

We note that the lattice parameter $\mathrm{a}_{0}$ tends to increase with temperature. This is due to the increase in lattice vibration because of the absorbed thermal energy. We also note that the hydrostatic pressure 
reduces the lattice parameter because it reduces vibrations of atomic lattice. The curve $V=f(T)$ increases moderately between $0 \mathrm{~K}$ and $100 \mathrm{~K}$ and is almost linear between $100 \mathrm{~K}$ and $800 \mathrm{~K}$.

The results of a study of heat capacity at a constant volume $-\mathrm{C}_{\mathrm{V}}$, the isotropy $-\mathrm{S}$ and the thermal expansion coefficient $-\alpha$ of CoGe compound under the effect of temperature between $0 \mathrm{~K}$ and $1200 \mathrm{~K}$ and under a hydrostatic pressure between $0 \mathrm{GPa}$ and $20 \mathrm{GPa}$ is presented in Fig. 9, Fig. 10 and Fig. 11.

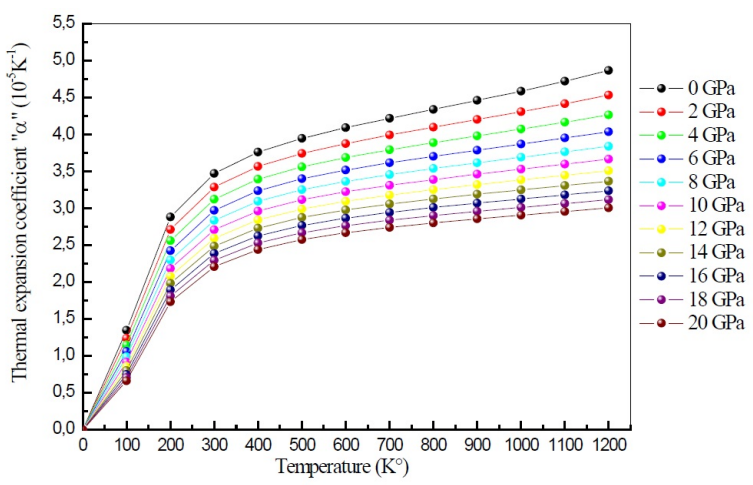

Fig. 9. Variation of the expansion coefficient $\alpha$ as a function of temperature for $\mathrm{CoGe}$ at different pressures.

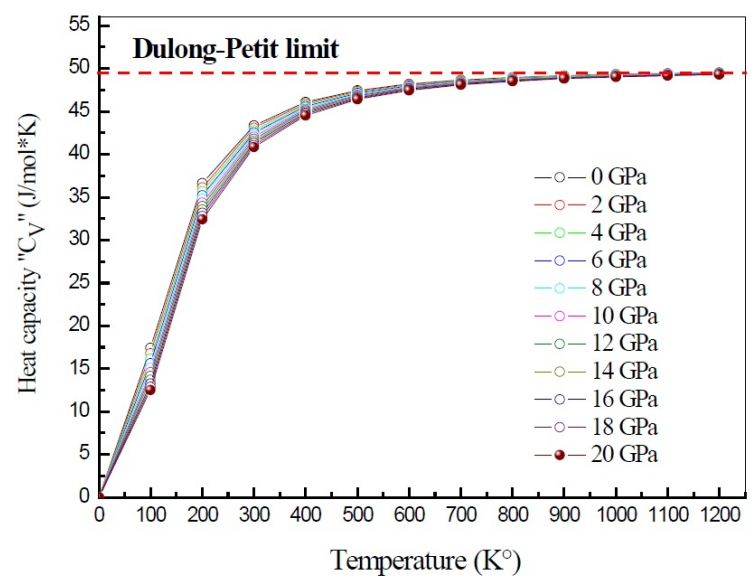

Fig. 10. Variation of the heat capacity $\mathrm{C}_{\mathrm{V}}$ as a function of temperature for $\mathrm{CoGe}$ at different pressures.

The thermal expansion coefficient $\alpha$ allows studying the expansion of a material under

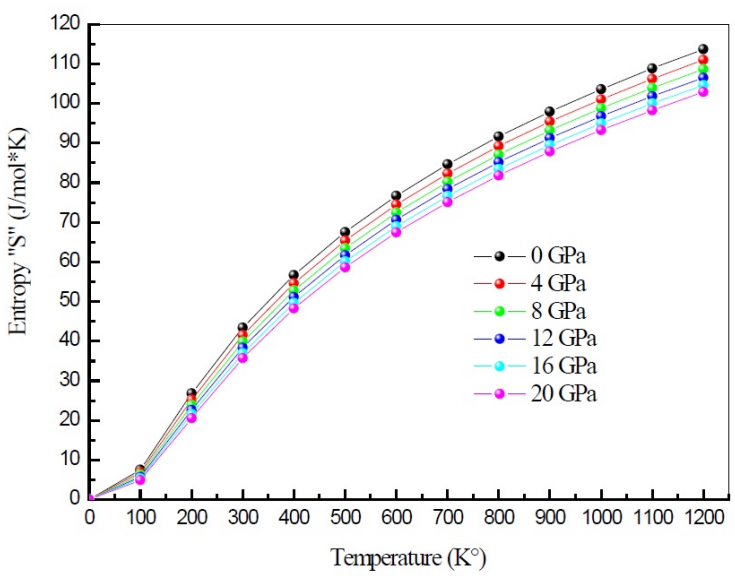

Fig. 11. Variation of the entropy $S$ as a function of temperature for $\mathrm{CoGe}$ at different pressures.

the effect of temperature (heating). From Fig. 9, it is seen that at low temperature, the thermal expansion is proportional to temperature changes and varies almost linearly while it increases moderately at high temperatures. For CoGe, the value of the coefficient of thermal expansion at room temperature for zero pressure is $\sim 3.47 \times 10^{-5} \cdot \mathrm{K}^{-1}$.

Fig. 10 presents the variation of heat capacity at a constant unit cell volume $-\mathrm{C}_{\mathrm{V}}$ under the effect of temperature and hydrostatic pressure for the CoGe compound. Note that at low temperature, heat capacity varies proportionally to $\sim \mathrm{T}^{3}$ and at high temperature it tends to the Dulong-Petit limit [52] $\left(\sim 3 \mathrm{NR}=49.51 \mathrm{~J} \cdot \mathrm{mol}^{-1} \cdot \mathrm{K}^{-1}\right)$. The calculated value of the heat capacity at room temperature for zero pressure is $\sim 43.345 \mathrm{~J} \cdot \mathrm{mol}^{-1} \cdot \mathrm{K}^{-1}$.

From Fig. 11, it is seen that the entropy increases with temperature which is due to the increase of the energy gained in the form of heat by $\mathrm{CoGe}$ compound. Its value at room temperature and zero pressure is $\sim 43,414 \mathrm{~J} \cdot \mathrm{mol}^{-1} \cdot \mathrm{K}^{-1}$.

We also noted that hydrostatic pressure tends to decrease the vibration of atomic lattice of solid materials; this can be reflected in a loss of energy. For a given temperature, when the pressure increases, the heat capacity at a constant volume $-\mathrm{C}_{\mathrm{V}}$, thermal expansion coefficient $-\alpha$ and entropy $-\mathrm{S}$ decrease. 


\section{Conclusions}

The work presented in this paper presents a complementary and comparative theoretical study with the works already done on the CoGe material in its B20 structure. This work focuses on the study of structural, elastic, electronic and thermal properties of $\mathrm{CoGe}$ compound. The results obtained in the structural part by the functional GGA-PBEsol are in a very good agreement with those previously found. Elastic constants have been predicted using two theoretical models: the Mehl's model and the model implemented in the IRelast package by Jamel. For this property, this work is the first that provides the values of elastic constants obtained by the two models which are similar to each other and confirm the mechanical stability of the compound in the CoGe B20 structure. According to the electronic part of this work, the CoGe compound has a semimetallic behavior. By the analysis of the electronic density, we have found that the nature of the bond between cobalt and germanium is of metalcovalent type. The effect of temperature and pressure on the behavior of the CoGe compound was also studied in this work by using the Debye model.

\section{Acknowledgements}

The author H. BOUAFIA kindly acknowledges Dr. Alberto Otero-de-la-Roza and Dr. Morteza Jamal for their help and support.

\section{References}

[1] KrajČí M., Hafner J., Phys. Rev. B, 87 (2013), 035436.

[2] Larchev V.I., Popova S.V., J. Less-Common Met., 87 (1982), 53.

[3] Takizawa H., Sato T., Endo T., Shimada M., $J$. Solid State Chem., 73 (1988), 40.

[4] Ditusa J.F., Zhang S.B., Yamaura K., Xiong Y., Prestigiacomo J.C., Fulfer B.W., Adams P.W., Brickson M.I., BRowne D.A., CAPAN C., FISK Z., Chan J.Y.,Phys. Rev. B, 90 (2014), 144404.

[5] Dong S.S., LiU S.G., TaO X.M., XIAO F.H., Huang L.H., YAng F., He Y., Chen Q., LiU H. S., Jin Z.P., Thermochim. Acta, 572 (2013), 94.

[6] Machado K.D., Gasperini A.A.M., Michielon DE Souza S., Maurmann C.E., Lima dE J.C., GRANDi T.A., Solid State Commun., 136 (2005), 466.

[7] Mikhaylushin A.S., Sato T., Carlson S., Simak S.I., HäUsserman U.N., Phys. Rev. B, 77 (2008), 014102.
[8] Ameereh G.I., Hamad B.A., Khalifeh J.M., Physica B, 403 (2008), 3503.

[9] Gazhulina A.P., Marychev M.O., J. Alloy. Compd., 623 (2015), 413.

[10] Kanazawa N., Charge and Heat Transport Phenomena in Electronic and Spin Structures in B20-type Compounds, Doctoral Thesis accepted by the University of Tokyo, Applied Physics Department, Tokyo, Springer Theses, 2015.

[11] Hsieh Y.F., Chen L.J., Marshall E.D., LaU S.S., Appl. Phys. Lett., 51(1987), 1588.

[12] Hsien Y.F., Chen L.J., Thin Solid Films, 162 (1988), 295.

[13] Madsen G.K.H., Blaha P., Schwarz K., Suöstedt E., Nordström L., Phys. Rev. B, 64 (2001), 195134.

[14] Schwarz K., Blaha P., Madsen G.K.H., Comput. Phys. Commun., 147 (2002), 71.

[15] BADER R.F.W., Atoms in Molecules: A Quantum Theory, Oxford University Press, Oxford, 1990.

[16] Bader R.F.W., Nguyen-Dang T.T., Tal Y., Rep. Prog. Phys., 44 (1981), 893.

[17] OTERO-DE-LA-RozA A., JohnSON E.R., LuAÑA V., Comput. Phys. Commun., 185 (2014), 1007.

[18] Otero-de-la-Roza A., Blanco M.A., Pendas M.A., LuAÑA V., Comput. Phys. Commun., 180 (2009), 157.

[19] Kokalu A., Comp. Mater. Sci., 28 (2003), 155.

[20] Bouafia H., Hiadsi S., Abidri B., Akriche A., Ghalouci L., Sahli B., Comp. Mater. Sci., 75 (2013), 1.

[21] Perdew J.P., Burke K., Ernzerhof M., Phys. Rev. Lett., 77 (1996), 3865.

[22] Perdew J.P., Ruzsinszky A., Csonka G.I., VyDrov O.A., Scuseria G.E., Constantin L.A., Zhou X., Burke K., Phys. Rev. Lett., 100 (2008), 136406.

[23] Hohenberg P., Kohn W., Phys. Rev. B, 136 (1964), 864.

[24] Hiadsi S., Bouafia H., Sahli B., Abidri B., Bouaza A., AKriche A., Solid State Sci., 58 (2016), 1.

[25] Blaha P., Schwarz K., Madsen G., KvasNICKA D., LUITZ J., WIEN2k, An Augmented Plane Wave + Local Orbitals Program for Calculating Crystal Properties, Karlheinz Schwarz, Techn. Universität Wien, Wien, 2001.

[26] Blaha P., Schwarz K., Sorantin P., Trickey S.K., Comput. Phys. Commun., 59 (1990), 339.

[27] Blochl P., Jepsen O., Andersen O.K., Phys. Rev. $B, 49$ (1994), 16223.

[28] Murnaghan F.D., P. Natl. Acad. Sci. USA, 30 (1944), 244.

[29] Wallace D.C., Thermodynamics of Cristals, Willey, New York, 1972.

[30] Jamal M., Asadabadi J.S., Ahmad I., Aliabad R.H.A., Comp. Mater. Sci., 95 (2014), 592. 
[31] IRelast Package Provided by JAMAL M. as a part of the commercial Code Wien2k, http://www.wien2k.at/ (2014).

[32] Mehl M.J., Phy. Rev. B, 47 (1993), 2493.

[33] Mehl M.J., Klein B.K., Papaconstantopoulos D.A., Intermetallic Compounds: Principle and Practice, in: Westbrook J.H., FleischeIR R.L. (Eds.), Principles, Vol. I, John Wiley and Sons, 1995.

[34] Voigt W., Lehrbush der Kristallphysik, Taubner B.B, Leipzig, 1928.

[35] Schreiber E., Anderson O.L., Soga N., Elastic Constants and Their Measurements, McGraw-Hill, New York, 1973.

[36] Roylance D., Mech. Prop. Mater, 2008.

[37] Pugh S.F., Philo. Mag., 45 (1954), 823.

[38] Mayer B., Anton H., Bott E., Methfessel M., Sticht J., Schmidt P.C., Intermetallics, 11 (2003), 23.

[39] Anderson O.L., J. Phys. Chem. Solids, 24 (1963), 909.

[40] Jamal M., Sarvestani K.N., Yazdani A., ReSHAK A.H., RSC Adv., 4 (2014), 57903.

[41] Wu X., Vanderbilt D., Hamann D.R., Phys. Rev. $B, 72$ (2005), 0355105.

[42] Anderson O.L., J. Phys. Chem. Solids, 12 (1959), 41.
[43] Pauling L., The Nature of Chemical Bond: An Introduction to Modern Structural Chemistry, Cornell University Press, Ithaca, 1960.

[44] Mori-SÁnchez P., Pendás M.A., Luaña V., J. Am. Chem. Soc., 124 (2002), 14721.

[45] Blanco M.A., Francisco E., Luaña V., Comput. Phys. Commun., 158 (2004), 57.

[46] Blanco M.A., Pendas M.A., Francisco E., ReCIO J.M., Franco R., J. Mol. Struc-Theochem., 368 (1996), 245.

[47] Florez M., Recio J.M., Francisco E., Blanco M.A., Martin Pendas A., Phys. Rev. B, 66 (2002), 144112.

[48] Francisco E., Recio J.M., Blanco M.A., Martin Pendas A., J. Phys. Chem. A, 102 (1998), 1595.

[49] Francisco E., Blanco M.A., Sanjurjo G., Phys. Rev. B, 63 (2001), 094107.

[50] POIRIER J.P., Introduction to the Physics of the Earth's Interior, Vol. 39, Cambridge University Press, Oxford, 2000.

[51] Hill R., Proc. Phys. Soc. London A, 65 (1952), 349.

[52] Petit A.T., Dulong P.L., Ann. Chim. Phys., 10 (1819), 395.

Received 2016-12-23

Accepted 2017-04-02 